\title{
Effect of Carbon Nanotube Dispersion on Electrochemical Behavior of the CNTs/LiCoO 2 Composite Cathode
}

\author{
Guo-ping Wang ${ }^{1, *}$, Mei Li $^{1}$, Yong Qian ${ }^{1}$, Li-fang Jia ${ }^{1}$, Yu-qiang Tao ${ }^{1, *}$, Jiang Qi $^{2}$, Xiaoying Lu ${ }^{2}$ \\ ${ }^{1}$ School of Chemistry and Chemical Engineering, University of South China, Hengyang 421001, PR \\ China \\ ${ }^{2}$ Key Laboratory of Advanced Technologies of Materials, Superconductivity and New Energy R\&D \\ Centre, Southwest Jiaotong University, Chengdu 610031, PR China \\ *E-mail: wgpcd@aliyun.com, taoyuqiang26@qq.com
}

doi: $10.20964 / 2021.07 .45$

Received: 15 June 2020 / Accepted: 16 March 2021 / Published: 31 May 2021

\begin{abstract}
Carbon nanotubes (CNTs) are utilized as conductive additives in lithium ion battery. Particularly, the effect of surfactant type on the CNTs' dispersion as well as correponding electrochemical behavior of $\mathrm{LiCoO}_{2}$ cathode is investigated. The results demonstrate that the dispersion of CNTs can be improved by both cationic and anionic surfactants, but the resulted electrodes show poor wettability with electrolyte. As a result, the electrochemical performance of corresponding $\mathrm{LiCoO}_{2}$-based cathode is worsened. Non-ionic polymer polyvinylpyrrolidone (PVP) act as an effective dispersant for promoting the dispersion of CNTs and improving the electrochemical performance of $\mathrm{LiCoO}_{2}$ cathode. In addition, CNTs after ball-milling show damaged structure and more agglomerates, which are not suitable for forming a continuous conductive network.
\end{abstract}

Keywords: Carbon nanotubes; Conductive additives; Dispersion; Lithium ion battery

\section{FULL TEXT}

(C) 2021 The Authors. Published by ESG (www.electrochemsci.org). This article is an open access article distributed under the terms and conditions of the Creative Commons Attribution license (http://creativecommons.org/licenses/by/4.0/). 